\title{
BI-ARC BOUNDARY FUNCTIONS ${ }^{1}$
}

\section{E. SNYDER}

Introduction. A function $f$ is a boundary function if $f$ is defined on the real line and is the limit in some restricted sense of a function $\Phi$ defined on the open upper half-plane or if $f$ is defined on the unit circle and is the limit in some restricted sense of a function $\Phi$ defined on the open unit disk. Bagemihl and Piranian [1] and Kaczynski [2] have studied boundary functions for the case where at each point of the boundary the limit of $\Phi$ is assumed to exist along some arc with one endpoint on the boundary. The author [5], [6] has studied boundary functions which are obtained by taking boundary limits relative to Stolz angles and approximate limits relative to Stolz angles. In this paper we assume that at each point of the boundary there are two arcs along which the limits of $\Phi$ exist and are equal.

We will let $R$ denote the set of real numbers.

Definition. If $x \in R$, an arc at $(x, 0)$ is a Jordan arc $\gamma$ with one endpoint at $(x, 0)$ and with all other points lying in the open upper half-plane.

Definition. $\Gamma$ is a bi-arc at $(x, 0)$ if $\Gamma$ is the union of two arcs at $(x, 0)$ which are disjoint except for the point $(x, 0)$.

Definition. Let $\Phi: R \times(0, \infty) \rightarrow R$ and $f: R \rightarrow R$. The function $f$ is a bi-arc boundary function for $\Phi$ if for each $x \in R$ there is a bi-arc $\Gamma_{x}$ such that $\lim \Phi(u, v)=f(x)$, as $(u, v) \rightarrow(x, 0)$, for $(u, v) \in \Gamma_{x}$.

Definition. Let $f: R \rightarrow R$. The function $f$ is of honorary Baire class two if there is a function $g: R \rightarrow R$ such that $g$ is of Baire class one and $f(x)=g(x)$ except on a countable set.

THEOREM 1. If a function $f$ is a bi-arc boundary function for a realvalued function $\Phi$ on the upper half-plane, then $f$ is of honorary Baire class two.

Proof. For the proof we will show that for any $\epsilon>0$ and any nonempty perfect set $P, P \subset R$, there are a point $x_{0} \in P$, a function $g: R \rightarrow R$ of Baire class one, and a neighborhood $(c, d)$ of $x_{0}$ such that $|f(x)-g(x)|<\epsilon$ for $x \in(c, d) \cap P$ except for countably many points. By a theorem of Lederer [3], this implies that there is a function $h$ of Baire class one which differs from $f$ on a countable set only.

Let $P$ be a nonempty perfect set of real numbers and let $\epsilon>0$ be

Presented to the Society, November 26, 1965; received by the editors August 5, 1966.

${ }^{1}$ Supported in part by NSF Institutional Grant GU-1514. 
given. For each $x \in R$, let $\Gamma_{x}$ denote the bi-arc at $(x, 0)$ along which the limit of $\Phi$ is equal to $f(x)$. Without loss of generality we may assume that all of the arcs involved intersect the line $y=1$. For any $r$, $1 \leqq r<\infty$, let

$$
\Gamma_{x}^{r}=\left\{(u, v) \in \Gamma_{x}: 0<v \leqq 1 / r\right\},
$$

and proceeding from the point $(x, 0)$ along the two arcs which constitute $\Gamma_{x}$, let $\gamma(x, r)$ and $\gamma^{\prime}(x, r)$ denote the first points which lie on the line $y=1 / r$.

Let $l(x, \theta)$ denote the half-line from $(x, 0)$ which lies in the upper half-plane and for which the angle between the $x$-axis and the halfline is $\theta, 0<\theta<\pi$. Then for each rational $\theta, 0<\theta<\pi$, and each natural number $n$, let

$$
\begin{aligned}
A_{n \theta}=\left\{x \in P:(u, v) \in \Gamma_{x}^{n} \Rightarrow\right. & |\Phi(u, v)-f(x)|<\epsilon / 4 \\
& \text { and } \left.l(x, \theta) \text { separates } \gamma(x, n) \text { and } \gamma^{\prime}(x, n)\right\} .
\end{aligned}
$$

It is easily seen that $P=\cup_{n=1}^{\infty} \bigcup_{\theta \text { rational; } 0<\theta<\pi} A_{n \theta}$. Since $P$ is of the second category in itself, it follows that there are a natural number $n_{0}$ and a rational number $\theta_{0}, 0<\theta_{0}<\pi$, such that $A_{n_{0} \theta_{0}}$ is of the second category in $P$. Then there is an open interval $I$ with $I \cap P$ nonempty such that $A_{n_{0} \theta_{0}}$ is dense in $I \cap P$ and such that $I \cap A_{n_{0} \theta_{0}}$ is of the second category in $P$. Let $x_{0}$ be a point of $A_{n_{0} \theta_{0}} \cap I$ which is a bilateral limit point of $A_{n_{0} 0_{0}} \cap I$. Project $\gamma\left(x_{0}, n_{0}\right)$ and $\gamma^{\prime}\left(x_{0}, n_{0}\right)$ to the $x$-axis parallel to the half-line $l\left(x_{0}, \theta_{0}\right)$ and denote by $(a, b)$ the open interval determined by this projection. Set $J=(a, b) \cap I$ and observe that if

$$
x \in A_{n_{0} \theta_{0}} \cap J, \quad \text { then } \Gamma_{x}^{n_{0}} \cap \Gamma_{x_{0}}^{n_{0}} \neq \varnothing .
$$

Denote by $\dot{P}$ the set $P$ minus the endpoints of the open intervals of the complement of $P$ and let $E$ be defined by

$$
E=\left\{z \in P \cap J: \Gamma_{z}^{n(z)} \cap \Gamma_{x}^{n_{0}}=\varnothing \text { for every } x \in A_{n_{0} \theta_{0}} \cap J\right\},
$$

where $n(z)$ denotes the larger of $n_{0}+1$ and the smallest natural number $n$ for which $(u, v) \in \Gamma_{z}^{n} \Rightarrow|\Phi(u, v)-f(z)|<\epsilon / 4$.

Assertion. The set $E$ is countable.

Suppose $E$ were uncountable. For each $z \in E$, the union of $\Gamma_{z}^{(z)}$ with the line segment $\{(z, y):-1 \leqq y \leqq 0\}$ is a triod. There can be only countably many disjoint triods in the plane (see $[4$, p. 254 , Theorem $75])$, hence there are $z_{1}, z_{2} \in E$ such that $\Gamma_{z_{1}}^{n\left(z_{1}\right)} \cap \Gamma_{z_{2}}^{n\left(z_{2}\right)} \neq \varnothing$. There is an $x \in A_{n_{0} \theta_{0}} \cap J$ with $x$ between $z_{1}$ and $z_{2}$. For this $x$, either $\Gamma_{z_{1}}^{n\left(z_{1}\right)} \cap \Gamma_{x}^{n_{0}}$ $\neq \varnothing$ or $\Gamma_{z_{2}}^{n\left(z_{2}\right)} \cap \Gamma_{x}^{n_{0}} \neq \varnothing$ which contradicts the fact that both $z_{1}$ and $z_{2}$ are in $E$. Therefore $E$ is countable. 
If $w \in \dot{P} \cap J$ such that $w \in E$, then there is a point $x_{1} \in A_{n_{0} \theta_{0}} \cap J$ such that $\Gamma_{w}^{n(w)} \cap \Gamma_{x_{1}}^{n_{0}} \neq \varnothing$. Let $\zeta \in \Gamma_{w}^{n(w)} \cap \Gamma_{x_{1}}^{n_{0}}$ and $\xi \in \Gamma_{x_{1}}^{n_{0}} \cap \Gamma_{x_{0}}^{n_{0}}$. It follows that

$$
\begin{aligned}
\left|f(w)-f\left(x_{0}\right)\right| \leqq & |f(x)-\Phi(\zeta)|+\left|\Phi(\zeta)-f\left(x_{1}\right)\right| \\
& +\left|f\left(x_{1}\right)-\Phi(\xi)\right|+\left|\Phi(\xi)-f\left(x_{0}\right)\right| \\
< &
\end{aligned}
$$

Define the function $g$ to have the value $f\left(x_{0}\right)$ for all $x$. For all points $w \in P \cap J$ except for those points belonging to the countable set $(P-\dot{P}) \cup E$, we have $|f(w)-g(w)|<\epsilon$. By Lederer's theorem, this implies that $f$ is of honorary Baire class two.

In [7] the author considered boundary functions which were obtained by limits relative to open sets with one point of the boundary of each open set being on the $x$-axis. However, the open sets were required to be "well behaved" in certain ways. In fact, it was pointed out that if each of the sets was merely an open set with connected closure, then the boundary function could be nonmeasurable. As a consequence of Theorem 1 above we have the following theorem in which the sets used are open connected sets.

TheоRem 2. Let $\Phi: R \times(0, \infty) \rightarrow R$. Suppose for each $x \in R$ there is an open connected set $G_{x} \subset R \times(0, \infty)$ such that $(x, 0)$ is an accessible boundary point of $G_{x}$ and $\lim \Phi(u, v)$, as $(u, v) \rightarrow(x, 0)$, exists if $(u, v)$ is restricted to lie in $G_{x}$. If $f: R \rightarrow R$ is the boundary function of $\Phi$ determined by the family of sets $\left\{G_{x}: x \in R\right\}$, then $f$ is of honorary Baire class two.

Bagemihl and Piranian [1] posed the following question. If $f$ is of Baire class two and is a boundary function for some continuous function on the open unit disk, is $f$ merely an honorary function of Baire class two? Kaczynski [2] answered the question in the affirmative. Theorem 2 of this paper provides another proof of Kaczynski's theorem.

Let $C$ denote the unit circle and $D$ denote the open unit disk.

Definition. Let $\Phi: D \rightarrow R$ and $f: C \rightarrow R$. The function $f$ is a boundary function for $\Phi$ if for each $p \in C$ there is an arc $\gamma$ at $p$ such that the limit of $\Phi$ as $q \rightarrow p$ along the arc exists and is equal to $f(p)$.

THEOREM 3. If $f$ is a boundary function for a continuous function $\Phi$ on the unit disk, then $f$ is an honorary function of Baire class two.

Proof. We observe first of all that Theorems 1 and 2 are valid in the context of the unit disk. For the proof we show that if $\Phi$ is con- 
tinuous and if the boundary limit of $\Phi$ exists along an arc, then the boundary limit of $\Phi$ exists for an open connected set with the boundary point as an accessible point.

Let $\gamma_{p}$ be an arc at $p \in C$ such that $\lim _{q \rightarrow p} \Phi(q)$ exists for $q \in \gamma_{p}$. Let $q \in \gamma_{p}, q \neq p$. Let $\eta(q)$ be the distance from $q$ to $C$. Since $\Phi$ is continuous at $q$, there is a positive number $\delta^{\prime}(q)$ such that $\left|q^{\prime}-q\right|<\delta^{\prime}(q)$ implies $\left|\Phi\left(q^{\prime}\right)-\Phi(q)\right|<\eta(q) / 2$. Let $\delta(q)$ be the minimum of $\delta^{\prime}(q)$ and $\eta(q) / 2$. Set $G_{p}=U_{q \in \gamma_{p}-\{p\}}\{s \in D:|s-q|<\delta(q)\}$. It is easily seen that $G_{p}$ is a connected open set and that $\lim _{s \rightarrow p} \Phi(s)$ exists for $s \in G_{p}$.

\section{REFERENCES}

1. F. Bagemihl and G. Piranian, Boundary functions for functions defined in a disk, Michigan Math. J. 8 (1961), 201-207.

2. T. J. Kaczynski, Boundary functions for functions defined in a disk, J. Math. Mech. 14 (1965), 589-612.

3. G. Lederer, Two theorems on Baire functions in separable metric spaces, Quart. J. Math. Oxford Ser. 11 (1960), 269-274.

4. R. L. Moore, Foundations of point set theory, Colloq. Publ., Vol. 13, Amer. Math. Soc., Providence, R. I., 1932.

5. L. Snyder, Continuous Stolz extensions and boundary functions, Trans. Amer. Math. Soc. 119 (1965), 417-427.

6. - Approximate Stolz angle limits, Proc. Amer. Math. Soc. 17 (1966), $416-422$.

7. - Stolz angle convergence in metric spaces, Pacific J. Math. 22 (1967).

UNIVERSITY OF VIRGINIA 